\title{
Uso do teste caixa e blocos na avaliação de destreza manual em crianças e jovens com síndrome de Down
}

\section{Box and block test in motor dexterity evaluation to children and young people with Down syndrome}

\author{
Renata Guimarães ${ }^{1}$, Silvana Maria Blascovi-Assis ${ }^{2}$
}

GUIMARÃES, R.; BLASCOVI-ASSIS, S. M. Uso do teste caixa e blocos na avaliação de destreza manual em crianças e jovens com síndrome de Down. Rev. Ter. Ocup. Univ. São Paulo, v. 23, n. 1, p. 98-106, jan./abr. 2012.

RESUMO: Este estudo objetivou avaliar a destreza manual de crianças e adolescentes com síndrome de Down (SD) nas idades de 7, 8, 9, 14 e 15 anos, utilizando o Teste de Caixa e Blocos (TCB). Participaram 50 crianças e jovens com SD, de ambos os sexos, que compuseram o grupo estudado (GSD), e 50 sem a síndrome, constituindo o grupo controle (GC). O teste foi aplicado individualmente em cada participante nas instituições colaboradoras no interior do Estado de São Paulo após aprovação do Comitê de Ética em Pesquisa da Universidade Presbiteriana Mackenzie. Os resultados indicaram que há desvantagem na destreza manual dos participantes com SD quando comparados ao GC. Observou-se também que não houve alteração estatisticamente significativa na destreza manual para o GSD entre as idades de 7 a 9 anos em comparação com as idades de 14 e 15 anos $(7 \mathrm{a}=30$ Blocos Por Minuto, 8a = 29 BPM, 9a =29 BPM), (14a=32 BPM, 15a = 34 BPM), evidenciando que quase não há evolução na destreza manual com a idade. O GC apresentou evolução de destreza manual proporcional à idade dos participantes $(7 \mathrm{a}=63 \mathrm{BPM}, 8 \mathrm{a}=66 \mathrm{BPM}$, $9 \mathrm{a}=68 \mathrm{BPM}, 14 \mathrm{a}=80 \mathrm{BPM}, 15 \mathrm{a}=81 \mathrm{BPM})$. Adicionalmente, foram observadas diferenças na forma de preensão dos blocos nos participantes do GSD, com a utilização da pinça entre os dedos polegar e médio em $36 \%$ dos casos contra 4\% no GC. Conclui-se que o Teste de Caixa e Blocos é uma opção eficiente e objetiva para a quantificação da destreza manual em populações com déficits intelectuais, pela fácil aplicação e por exigir pouca compreensão dos avaliados.

DESCRITORES: Destreza motora; Síndrome de Down; Crianças com deficiência; Habilidades para realização de testes.

\footnotetext{
1. Terapeuta Ocupacional, Mestre em Distúrbios do Desenvolvimento pela Universidade Presbiteriana Mackenzie.

2. Fisioterapeuta, Docente do Programa de Pós-Graduação em Distúrbios do Desenvolvimento da Universidade Presbiteriana Mackenzie. Endereço para correspondência: Renata Guimarães. Rua Marechal Castelo Branco, 475. 18031-300 Sorocaba, SP, Brasil. E-mail: re_ge@hotmail.com
} 


\section{INTRODUÇÃO}

Síndrome de Down (SD) é a síndrome ge-
nética mais frequente associada ao déficit
intelectual. Sua incidência é de um a dois indivíduos a cada mil crianças nascidas vivas, tendo relação direta com a idade materna (SCHWARTZMAN, 1992). Os dados epidemiológicos brasileiros indicam que a incidência é de 1:600 nascidos vivos (GARCIAS et al., 1995).

Dentre as múltiplas alterações físicas que ocorrem na SD, em cerca de 70 a $80 \%$ dos casos as alterações encontradas no membro superior são: membros curtos, mãos curtas e largas, dedos curtos; prega palmar transversal única (40 a 50\%); clinodactilia no quinto dedo em 50 a $60 \%$ dos casos, provavelmente pela displasia ou ausência da falange média (OTTO et al., 2004).

A diminuição do tônus muscular é um dado altamente consistente que auxilia no diagnóstico, sendo uma das causas do atraso e dificuldade na realização de movimentos adequados dentro do desenvolvimento motor normal. A hipotonia interferirá no desenvolvimento motor da criança com SD, atrasando a aquisição de habilidades, a exploração do ambiente, causando déficits sensoriais e vivenciais, refletidos também no desempenho cognitivo (GUSMAN; TORRE, 1999). Quanto ao desenvolvimento de habilidades motoras, as crianças com SD, comparativamente às crianças com desenvolvimento normal, passam por todas as etapas, porém, apresentam atraso e lentidão nas aquisições do padrão motor básico (MANCINI et al., 2003; GIMENEZ et al., 2004; NUNES; DUPAS, 2011).

A hipotonia interfere diretamente na aquisição de habilidades do desenvolvimento motor da criança com SD, limitando as habilidades físicas de coordenação motora, tanto grossa como fina. A hipotonia gera o aumento da flacidez, hipermobilidade articular, com hiperextensão e frouxidão ligamentar e muscular, o que causará diminuição dos movimentos e dificuldade em realizá-los, atrasando por consequência habilidades como controle da cabeça, sentar e manipular objetos (GUSMAN; TORRE, 1999). Godoy e Barros (2005) encontraram força preensora diminuída em crianças com SD em seu estudo, quando comparadas a crianças saudáveis.

A mão é uma importante ferramenta criativa, uma extensão do intelecto, servindo como meio para a comunicação não verbal, e o mais importante órgão sensorial tátil. A mão é capaz de realizar movimentos extremamente finos e sensíveis, assim como executar tarefas que requerem grande força. A qualidade e o desempenho nas atividades da vida diária relacionadas ao trabalho e às atividades de lazer são determinados em grande parte pelas funções da mão e pela destreza manual (CARMELI et al., 2003). A destreza manual é frequentemente avaliada na terapia ocupacional para medir o déficit motor, assim como monitorar sua evolução e medir o grau de recuperação alcançado pelos pacientes quanto ao potencial para retornar ao trabalho (DESROSIERS et al., 1994; POIRIER, 1988).

Poirier (1988) relaciona a destreza manual a aprendizagem e treino, definindo-a como uma habilidade manual que requer coordenação rápida dos movimentos voluntários grossos ou finos, baseada em capacidades desenvolvidas através de aprendizagem, treinamento e experiência. Desrosiers (1997) divide o conceito de destreza manual em fina e grossa. Destreza fina ou digital é a capacidade de realizar movimentos rápidos e controlados, e manipular habilmente pequenos objetos com rapidez e precisão, utilizando principalmente as extremidades distais dos dedos. Destreza grossa, ou simplesmente destreza manual é a habilidade de utilizar mão e braço em movimentos controlados, na manipulação de objetos maiores (FLEISHMAN et al., 1962). Portanto, a destreza manual em seu sentido mais amplo depende de ambas as habilidades, fina e grossa, assim como da coordenação e agilidade adquiridas através da prática de atividades envolvendo a manipulação de objetos (DESROSIERS et al., 1994).

O Teste de Caixa e Blocos (TCB) foi criado para ser um teste pré-vocacional para pessoas com deficiência física. Permite avaliar e medir de forma simples a destreza manual de pacientes com problemas neuromusculares e paralisia cerebral. Foi idealizado e registrado em 1957 por A. J. Ayres e P. H. Buehler (MATHIOWETZ et al., 1985). Desrosiers et al. (1994) consideraram o TCB um teste de avaliação rápida, simples e confiável para a mensuração da destreza manual em pessoas idosas. O TCB foi validado no Brasil pelo Grupo de Neurologia da Santa Casa de São Paulo, em um estudo com 446 indivíduos (207 homens e 239 mulheres, com idades variando entre 15 e 86 anos) portadores de esclerose múltipla e controles. Os dados obtidos para sujeitos normais foram semelhantes aos originalmente descritos por Mathiowetz et al. em 1985 (MENDES et al., 2001). Nunes (2007) considera o TCB o teste mais simples e popular de função manual.

A escolha do TCB para esta pesquisa foi feita criteriosamente, considerando o público com SD a ser avaliado. Não se trata apenas de quantificar a destreza manual, mas em função do déficit intelectual das pessoas com SD, foi importante identificar um teste de fácil compreensão para que o intelecto dos avaliados não comprometesse o seu entendimento e realização, e assim não alterar os resultados finais. 


\section{OBJETIVOS}

Avaliar e comparar o desempenho em destreza manual, de crianças com e sem SD utilizando o teste de destreza manual Caixa e Blocos e verificar o efeito da idade na evolução da destreza.

\section{PROCEDIMENTOS METODOLÓGICOS}

Participaram deste estudo 100 crianças e adolescentes na faixa etária de 7 a 9 anos e 14 e 15 anos, de ambos os sexos, sendo 50 com síndrome de Down (grupo SD - GSD) e 50 sem a síndrome (grupo controle - GC). O estudo foi avaliado e aprovado pelo Comitê de Ética em Pesquisa da Universidade Presbiteriana Mackenzie (processo CEP/UPM $n^{\mathrm{o}}$ 1068/08/2008, CAAE no 0046.0.272.000-08).

Foram critérios de exclusão para ambos os grupos o diagnóstico de doença ou distúrbio incapacitante ou limitante de função em membros superiores e o uso regular de medicação que pudesse comprometer as funções motoras. Também foram excluídos os participantes que demonstraram dificuldade de compreensão para realização do teste. Assim, excluíram-se do estudo duas crianças com SD cujo desempenho cognitivo não permitiu a compreensão da tarefa. Dessa forma finalizaram o estudo 50 participantes com SD e 50 sem a síndrome (Tabela 1).

Tabela 1. Distribuição da amostra estudada por idade e sexo

\begin{tabular}{l|l|l|l|l|l}
\hline Idade (anos) & \multicolumn{2}{|c|}{ GSD } & \multicolumn{2}{c|}{ GC } & TOTAL \\
\hline & 0 & + & 0 & + & \\
\hline 7 & 5 & 5 & 4 & 6 & 20 \\
\hline 8 & 6 & 4 & - & 10 & 20 \\
\hline 9 & 6 & 4 & 5 & 5 & 20 \\
\hline 14 & 4 & 6 & 6 & 4 & 20 \\
\hline 15 & 7 & 3 & 6 & 4 & 20 \\
\hline TOTAL & 28 & 22 & 21 & 29 & 100 \\
\hline
\end{tabular}

Locais de realização da pesquisa: As crianças e adolescentes com SD foram contatadas em instituições especializadas e escolas regulares localizadas no interior do Estado de São Paulo, nas cidades de Sorocaba, Campinas, Pilar do Sul, Piedade e São Roque. Outras cidades foram contatadas, porém não houve possibilidade de coleta de dados pela indisponibilidade em receber a pesquisadora ou por não estarem matriculadas crianças e jovens na idade do estudo. A coleta de dados nas cinco cidades ocorreu em 13 locais diferentes, nas próprias instituições, após a leitura da carta de informação à instituição e aos pais ou responsáveis dos sujeitos da pesquisa, quando receberam todas as informações sobre o estudo (objetivos e procedimentos) e assinaram o Termo de Consentimento Livre e Esclarecido.

Procedimentos: A avaliação dos sujeitos da pesquisa foi feita através do TCB, por ser um instrumento de fácil aplicação e cuja utilização pode ser repetida quantas vezes forem necessárias até a uniformização das condições de aplicação do teste.

O TCB é utilizado para medir a destreza manual grossa e aplicado de forma individual, permite a observação e medida de tempo e resistência ao realizar a tarefa de transferência dos blocos. É considerado de fácil confecção e aplicação. Na avaliação da destreza da mão e dos dedos, o TCB possibilita medir a velocidade de manipulação dos objetos e observar a qualidade de função manual (NUNES, 2007). Foi caracterizado e descrito por Elui e Fonseca (2003) como sendo um teste confiável, pois no teste-reteste mantém o resultado anterior, permite amostra de normalidade para estabelecer o comparativo, quantifica a destreza dos dedos e habilidade bimanual. Por ser um teste essencialmente motor, o TCB não avalia o padrão intelectual e para sua execução é necessária a compreensão apenas de ordens simples na realização da manobra solicitada. Não há complexidade nas solicitações e ordens que as crianças com SD a serem avaliadas terão que realizar.

A caixa de madeira utilizada no TCB foi confeccionada seguindo as indicações propostas no teste, com as dimensões externas (padronizadas): $53,7 \mathrm{~cm}$ de comprimento, $25,4 \mathrm{~cm}$ de largura e bordas com 7,5 cm de altura, com uma divisória central vertical de $15,2 \mathrm{~cm}$ de altura separando a caixa em dois compartimentos de tamanhos iguais. Esta divisória contém dois orifícios para facilitar o transporte da caixa. A espessura da madeira utilizada na construção foi de $1,0 \mathrm{~cm}$. Cento e cinquenta blocos de madeira em forma de cubos com 2,5 cm de lado foram pintados nas cores primárias (vermelho, azul e amarelo), sendo 50 unidades de cada cor (MATHIOWETZ et al., 1985).

Foi necessário vedar os orifícios da divisória central, pois na primeira aplicação do teste a criança com SD tentou passar o bloco por essa abertura. Como a função dos orifícios é apenas facilitar o transporte da caixa de TCB, concluiu-se que sua oclusão não alteraria o resultado do teste, evitando repetição do fato.

Foram observadas as indicações de Mendes et al. (2001) para a aplicação do teste (os participantes não demonstraram estar sonolentos, irritados, com fome ou qualquer estado que pudesse interferir nos resultados). Considerou-se o interesse, participação e compreensão dos 
avaliados. Para manter a atenção dos sujeitos de pesquisa, a contagem dos blocos transportados foi feita em voz alta pelo examinador. Os testes foram realizados em ambiente sem ruídos e bem iluminado, com o participante sentando confortavelmente em cadeira adequada ao seu tamanho, com a caixa colocada à sua frente, em posição horizontal, com a divisória alinhada com o plano sagital da cabeça do participante, permitindo assim total visão da área do equipamento usado para sua avaliação de destreza manual.

Figura 1. Caixa de madeira utilizada no Teste de Caixa e Blocos

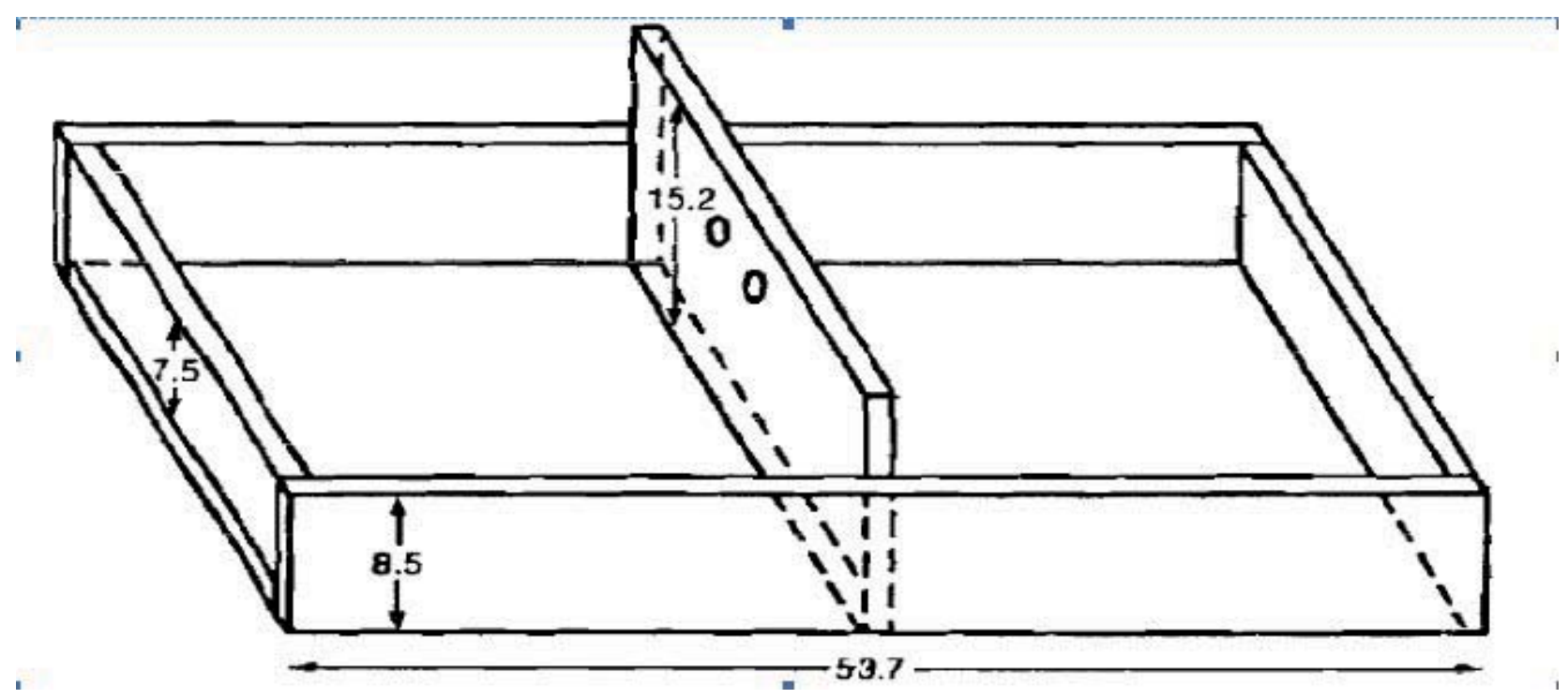

No início do teste, o participante recebeu a orientação de que deveria passar um bloco de cada vez para o outro compartimento da caixa, começando com a mão dominante. Todos os participantes puderam treinar por 15 segundos antes da aplicação do teste. Após isso os blocos foram todos colocados no lugar inicial.

A medida do teste foi expressa pelo número de blocos transferidos em um minuto (BPM) de um compartimento para o outro da caixa. O tempo de um minuto foi marcado por cronômetro (Speedo sporty), contabilizando-se o desempenho (número de blocos transferidos de um lado para outro na caixa) aos 30 e aos 60 segundos do início do teste. $\mathrm{O}$ número de blocos transferidos foi anotado na ficha de avaliação (Figura 2). O examinador identificou previamente o lado dominante dos participantes, e anotou igualmente na folha de resultados.

Os resultados e os dados de identificação do participante foram anotados em ficha individual como Avaliação $\mathrm{n}^{\circ} 1$. Depois deste procedimento, foi feito o teste-reteste com o mesmo examinador, onde foi repetido o processo da primeira avaliação, denominada Avaliação n ${ }^{\circ} 2$.

Análises estatísticas: Os dados coletados foram organizados em uma planilha Excel ${ }^{\circledR}$ e analisados com o programa Statistical Package for the Social Science SPSS $®$ versão 13.0. Foi utilizado o teste de Shaphiro Wilk para avaliar a normalidade das variáveis quantitativas e uma vez que não foi encontrada normalidade da amostra foi aplicado o Teste $\mathrm{H}$ de Kruskall-Wallis para comparar as medidas entre três ou mais amostras independentes. Para todas as análises adotou-se o nível de significância de 0,05 .

\section{RESULTADOS}

Os dados da comparação do desempenho entre os grupos GSD e GC evidenciaram melhores resultados no GC, ou seja, as crianças sem SD obtiveram melhor pontuação com o aumento da idade e no grupo das crianças com SD essa diferença não foi significativa, conforme o gráfico 1 .

Quando comparados por faixas de idade (Faixa $1=$ 7 a 9 anos, Faixa $2=14$ e 15 anos) observa-se que houve evolução na destreza manual, representada pelo número de blocos transferidos para GC, não ocorrendo o mesmo para GSD, conforme o gráfico 2. Observa-se melhor desempenho inicial e final para GC. 
Gráfico 1. Desempenho dos grupos GSD e GC por idade

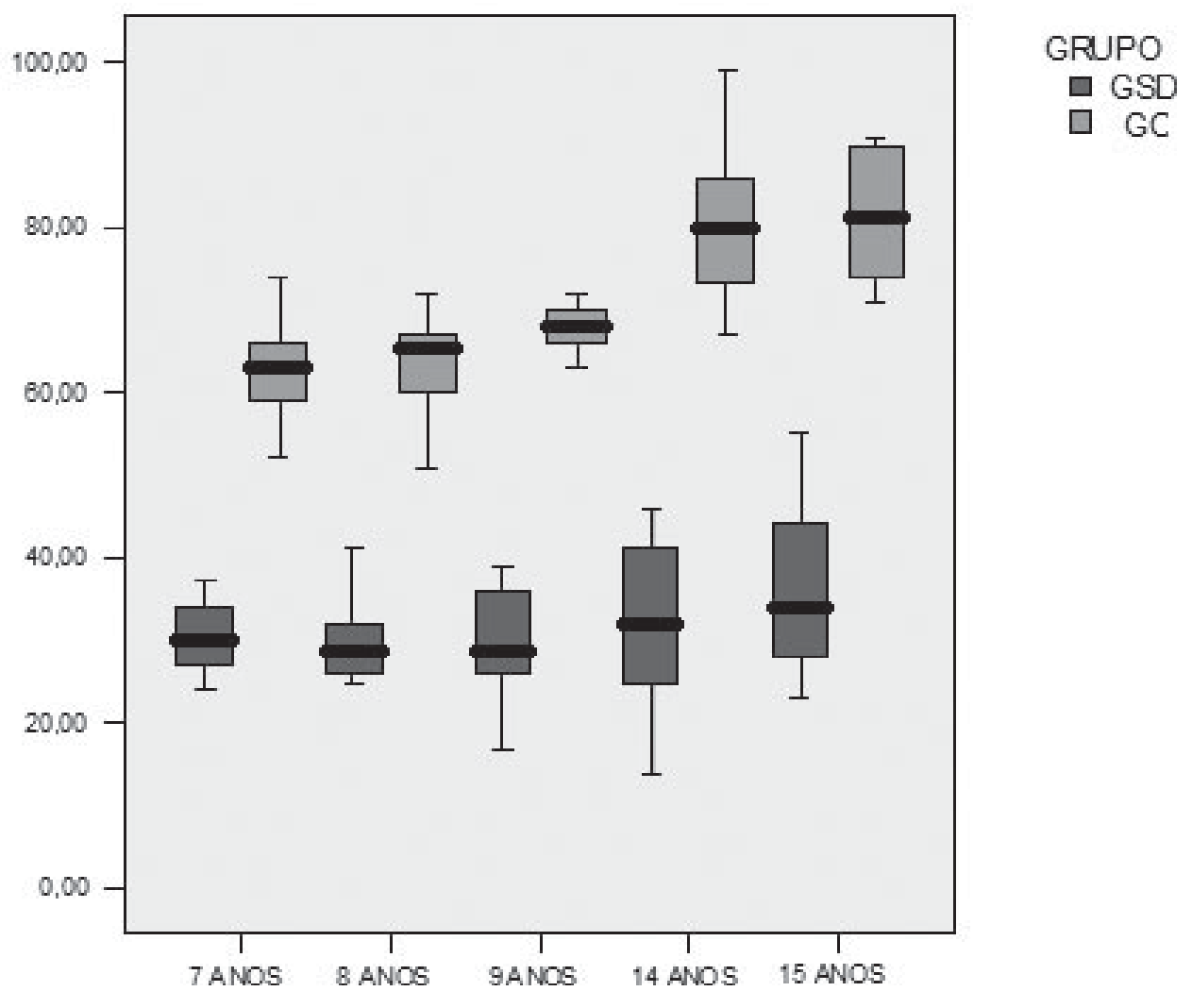

Gráfico 2. Comparação do desempenho entre GSD e GC em blocos por idade

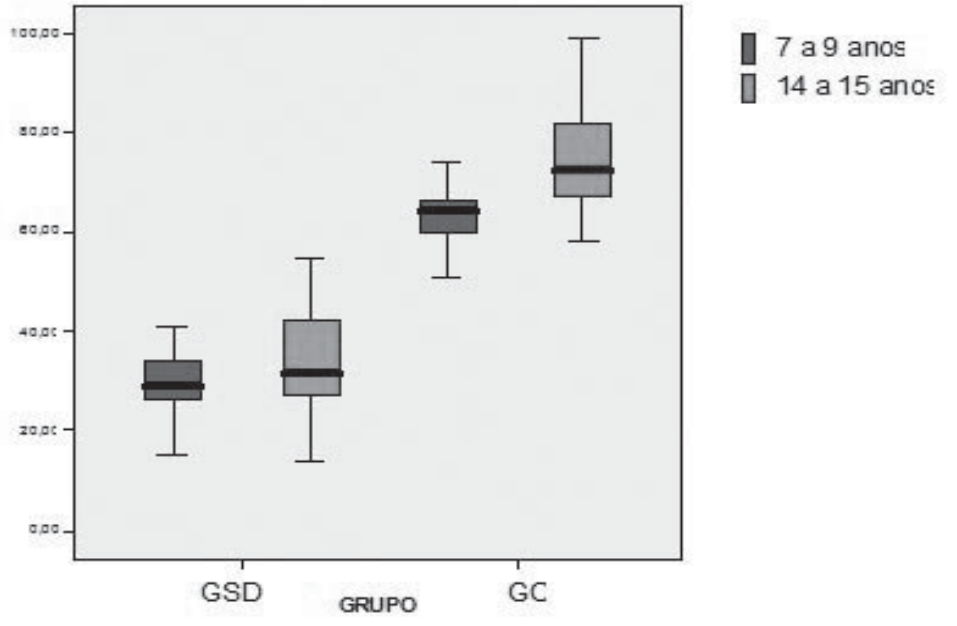


Os resultados do teste de Kruskal-Wallis indicam que há diferenças para a melhor resposta segundo a idade para $\mathrm{GC}$, e pode-se verificar que há diferenças entre as respostas obtidas de sujeitos com 7 e 8 anos, com 7 e 14 anos e ainda com 7 e 15 anos. As respostas obtidas para 8 anos diferem das respostas obtidas para 14 anos e das obtidas para 15 anos (Tabela 2).

Tabela 2. Resultados da comparação intragrupo da melhor resposta por idade

\begin{tabular}{l|l|l|l}
\hline $\begin{array}{l}\text { G r u p o } \\
\text { (anos) }\end{array}$ & Idade & Mediana $(\mathrm{P} 25 ; \mathrm{P} 75)$ & \\
\hline GSD & 7 & $30(26,25 ; 34,75)$ & $\mathrm{p}=0,513$ \\
\hline & 8 & $29(25,75 ; 33,75)$ & \\
\hline & 9 & $29(25,75 ; 36,75)$ & \\
\hline & 14 & $32(24,75 ; 41)$ & \\
\hline & 15 & $34(27,75 ; 45)$ & \\
\hline GC & $7 \mathrm{a}$ & $63(59 ; 66,25)$ & $\mathrm{p}=0,000002$ \\
\hline & 8 & $66(59,75 ; 68)$ & \\
\hline & 9 & $68(65,25 ; 70,5)$ & \\
\hline & $14 \mathrm{a}$ & $80(71,75 ; 86,5)$ & \\
\hline & $15 \mathrm{a}$ & $81(73,75 ; 90)$ & \\
\hline
\end{tabular}

a diferenças significativa $\mathrm{p}<0,05$.

Durante a aplicação do teste foi observado que algumas crianças apreendiam o bloco usando pinça entre o polegar e o dedo médio. Esse padrão diferenciado de pinça passou a ser anotado durante a execução da tarefa de transferência dos blocos. Como ele se repetiu diversas vezes entre o grupo de crianças e adolescentes com SD foi contabilizado para ser comparado posteriormente ao grupo controle. Constatou-se que dentre as 50 crianças com SD avaliadas, 18 participantes realizaram essa pinça entre $1^{\mathrm{a}} \mathrm{e}$ $3^{a}$ falange, sendo que no grupo controle apenas 02 participantes executaram esse mesmo padrão.

\section{DISCUSSÃO}

O desempenho de ambos os grupos (GSD e GC) apresentou melhores resultados na segunda avaliação (Avaliação $\mathrm{n}^{\circ}$ 2) em função da maior familiaridade dos sujeitos avaliados com o material apresentado. Observou-se também melhor desempenho para o lado dominante, resultado que concorda com o estudo de Mathiowetz et al. (1985).

Através dos dados obtidos pelo TCB, constatou-se a pouca evolução na destreza manual entre o grupo com SD de 7 a 9 anos e os adolescentes de 14 e 15 anos. En- quanto a média da diferença entre o grupo de idade menor e o grupo de adolescentes foi de aproximadamente de 2 a 5, no GC a diferença das médias entre o grupo menor e os adolescentes foi em torno de 12 a 18 blocos, variação bastante significativa.

As avaliações transcorreram conforme o planejamento apresentado no método, e embora Cooper (2001) oriente os profissionais que desejam fazer uso do TCB a repetir as medidas do instrumento de acordo com as especificações publicadas no texto original de Mathiowetz et al. (1985), foi necessária uma adaptação na configuração do material do teste (vedação dos orifícios na divisória central) e alteração na aplicação do teste (contagem em voz alta) conforme relatado acima. Acredita-se que essas adaptações não interferiram na execução da tarefa e nem nos resultados, tornando o TCB mais preciso e claro para as pessoas com déficit intelectual. Outros autores também fizeram alterações nesse instrumento como Guzzo (2008) e Garros et al. (2010), que vedaram os orifícios e trocaram os blocos por cilindros ao utilizar o teste com pacientes hemiplégicos. Essas adaptações tornaram o teste mais viável a esse grupo.

O TCB foi aplicado inicialmente no GSD e posteriormente no GC, sendo que a contagem em voz alta foi mantida na avaliação de todos os participantes. Esse procedimento mostrou-se eficaz ao melhorar o controle do número de blocos transferidos aos 30 e 60 segundos. Ferrigno (2007) salienta que na situação de avaliação de crianças, o examinador deve adaptar a linguagem facilitando sua compreensão, ao mesmo tempo em que deve estar atento para interpretar as respostas o mais fidedignamente possível.

Os dados apresentados nas análises estatísticas mostram que existe defasagem na destreza manual das crianças menores (de 7 a 9 anos) quando comparadas com o grupo controle de mesma idade. Esse dado concorda com estudos e citações de autores que, ao se referirem à mobilidade manual do indivíduo com SD, relatam alterações motoras (LATASH et al., 2002) e dificuldades da capacidade manipulativa e função preensora, observadas durante a exploração de objetos (GUSMAN; TORRE, 1999). Porém como essas alterações não foram quantificadas, não era possível medir ou calcular a defasagem. Ao estudar as medidas apresentadas pelo teste de destreza manual TCB, tornou-se possível comparar quantitativamente a defasagem do grupo de crianças e adolescentes com SD com o grupo de desenvolvimento típico.

As medidas obtidas das transferências de BPM dos 60 segundos do lado dominante, Avaliação $\mathrm{n}^{\circ} 2$ do GC, apresentam diferença bastante significativa em relação às medidas correspondentes do GSD, chegando a ser mais 
que o dobro em todas as idades. Este desempenho prejudicado na destreza manual do grupo estudado pode ter sido influenciado por fatores diversos como: o déficit intelectual (SCHWARTZMAN, 1992), a hipotonia peculiar da síndrome (GUSMAN; TORRE, 1999), as múltiplas alterações físicas como membros curtos, mãos curtas e largas, dedos curtos; prega palmar transversal única (OTTO et al., 2004) ou dificuldades na coordenação de movimentos (LATASH et al., 2002). Essas hipóteses surgem como incentivo para estudos mais aprofundados sobre o tema, visando sempre privilegiar para as crianças e adolescentes com SD um acompanhamento terapêutico estruturado em pesquisas que mostrem não apenas os déficits, mas que identifiquem as causas dos problemas apontados, visando também uma forma de prevenção ou benefício na abordagem. A descoberta de um padrão de pinça diferenciado realizado por $36 \%$ das crianças do grupo com $\mathrm{SD}$, significativamente maior em relação aos 4\% do grupo controle, é um dado relevante a ser pesquisado posteriormente em relação à causa, importância e necessidade de abordagem terapêutica. Esse achado pode estar relacionado à característica anatômica da mão e dedos na SD ser menor que as dimensões de indivíduos normais. Apesar deste padrão de pinça polegar e médio não ser usual, observou-se sua utilização de forma funcional pelas crianças no teste TCB.

$\mathrm{Na}$ literatura pesquisada não foram encontradas referências sobre o uso de pinça entre o primeiro e terceiro dedos. Meyerhof (1994) cita que durante a pinça o dedo médio fica disposto ao lado do quarto e quinto dedos sem participar ativamente da oponência. Gimenez et al. (2004) sugerem que indivíduos com SD recorrem a estratégias diferenciadas para solucionar dificuldades motoras, quando comparados a pessoas sem SD. Outros estudos investigando este tipo de manuseio pelas crianças com SD poderão contribuir para maior conhecimento dos modelos de preensão funcional característico deste grupo.

A constatação desta diferença no tipo de pinça revela que o TCB foi sensível para detectar alterações no tipo de preensão, mesmo não sendo esse o objetivo principal dentro da avaliação de destreza manual, conforme referido por Nunes (2007).

Dentre as dificuldades motoras apresentadas, desde o padrão hipotônico ou falta de coordenação digital próprias do déficit de força ou agilidade, pergunta-se qual o motivo para esse padrão de pinça entre polegar e médio, ou a sua relevância, já que a criança desempenha a atividade de modo funcional. Como já citado por Latash et al. (2002), as pessoas com SD em geral realizam movimentos semelhantes ao grupo de normalidade, porém à sua própria maneira, podendo ser vistos pelos outros como diferentes ou desajeitados. Todas essas dúvidas surgem como sugestão para outros estudos.

Alguns instrumentos de avaliação possibilitam que seus componentes sejam usados como técnicas terapêuticas durante o tratamento, auxiliando a realização do treino e a melhora do ponto identificado como deficitário durante o teste, portanto não apenas como conteúdo avaliativo (ELUI; FONSECA, 2003; FERRIGNO, 2007). Durante a utilização do TCB nesta pesquisa e constatou-se que devido a sua simplicidade e facilidade de aplicação, esse material pode muito bem ser utilizado em terapia, como proposta terapêutica visando estimular e melhorar a destreza manual. Magalhães (1997) concorda que a avaliação é o primeiro momento de contato com o paciente e um importante instrumento para captar os progressos durante o tratamento, comparar a eficiência dos vários tipos de abordagens terapêuticas e finalmente documentar a evolução de forma eficaz, concreta e objetiva. O TCB mostra-se adequado a essas propostas.

A pesquisa sobre um teste adequado e a consequente seleção do Teste Caixa e Blocos para avaliação da destreza manual no indivíduo com SD é um primeiro passo para mostrar a necessidade de ênfase na avaliação da função e coordenação motora de membros superiores desta população e incentivar a busca de testes mais precisos sobre preensão e pinça em futuros estudos.

Optou-se por não considerar possíveis diferenças de gênero para os resultados do teste, considerando que no estudo de Mendes et al. (2001), o uso do teste não detectou diferenças em relação ao sexo. Também não foi realizada a correlação com o tamanho das mãos dos avaliados, considerando que diversos autores já comprovaram as diferenças anatômicas presentes no grupo de crianças com $\mathrm{SD}$. Sugere-se que em futuros estudos, as influências das medidas da mão sejam consideradas com populações com ou sem SD.

\section{CONCLUSÕES}

A avaliação da destreza manual com o TCB constatou diferenças importantes no desempenho de crianças e adolescentes com SD quando comparados ao grupo controle, observando-se desempenho inferior para o GSD. Foi possível, através do TCB, observar melhora no desempenho de acordo com a idade para o GC e estabilização dos resultados nas duas faixas etárias estudadas para o GSD, indicando pouca evolução na destreza manual com o passar dos anos para esse grupo. Observou-se ainda o uso de pinça entre o primeiro e o terceiro dedos para $36 \%$ do GSD, indicando estratégias diferenciadas por parte desse grupo para executar a tarefa. Ressalta-se a necessidade de outros estudos que tragam mais detalhes sobre atividades de coordenação ma- 
nual envolvendo destreza, força de preensão e coordenação manual na execução de tarefas diversas a fim de ampliar o conhecimento sobre o desempenho de pessoas com SD nesse tipo de habilidade.

GUIMARÃES, R.; BLASCOVI-ASSIS, S. M. Box and block test in motor dexterity evaluation to children and young people with Down syndrome. Rev. Ter. Ocup. Univ. São Paulo, v. 23, n. 1, p. 98-106, jan./abr. 2012.

\begin{abstract}
The aim of this study was to evaluate manual dexterity of children and adolescents $(7,8,9,14$ and 15 years) with Down syndrome (DS), using the Box and Block Test (BBT). Fifty children and adolescents of both genders with Down syndrome (DS group - DSG) and 50 normal participants (control group - CG) took part in the study. The BBT was applied individually to each participant in his/hers own school or institution, in cities of São Paulo state, after approval of the Ethics Committee, from the Universidade Presbiteriana Mackenzie. The results showed a drawback in the manual dexterity in the DSG when compared to CG. There was no statistically significant variation on the manual dexterity of DSG between ages 7 to 9 years compared to 14 and 15 years $(7 \mathrm{y}=30$ Blocks Per Minute, $8 y=29$ BPM, 9y = 29 BPM $) ;(14 y=32$ BPM, 15y = $34 \mathrm{BPM})$, revealing that there is almost no improvement in manual dexterity related to age in this group. Conversely, in CG an age-dependent improvement was observed (7y $=63 \mathrm{BPM}, 8 \mathrm{y}=66$ BPM, 9y $=68$ BPM, 14y $=80$ BPM, 15y = 81 BPM). Moreover, differences were observed in the way participants of DSG grip the blocks, using thumb and middle finger (36\%) versus $4 \%$ in CG. We conclude that the Box and Block Test is an objective and efficient option to quantify manual dexterity of intellectually drawback population for its fast and qualified information, and for the easiness of application and comprehension by the participants.
\end{abstract}

KEYWORDS: Motor skills; Down syndrome, Disabled children; Test taking skill.

\title{
REFERÊNCIAS
}

1. CARMEli, E.; PATISH, H.; COLEMAN, R.: The Aging Hand. J. Gerontol. Med. Sci., v. 58A, n. 2, p. 146-152, 2003.

2. COOPER, C. Hand impairments. In: Trombly, C.A.; Radomski, M.V. Occupational Therapy for Physical Dysfunction. $5^{\text {th }}$ ed. Baltimore: Lippincott Williams \& Wilkins, 2001.

3. DESROSIERS, J.; BRAVO, G.; HÉBERT, R.; DUTIL, E.; MERCIER, L. Validation of the Box and Block Test as a measure of dexterity of elderly people: reliability, validity, and norms studies. Arch. Phys. Med. Rehabil., v. 75, n. 7, p. 751-755, 1994.

4. DESROSIERS, J.; ROCHETTE, A.; HÉBERT. R.; BRAVO, G.: The Minnesota Manual Dexterity Test: Reliability, validity and reference values studies with healthy elderly people. Can. J. Occup. Ther., v. 64, n. 5, p. 270-276, 1997.

5. ELUI, V. M. C.; FONSECA, M. C. R. Destreza. In: ARAÚJO, P. M. P. (Org). Recomendações para avaliação do membro superior. São Paulo: SBTM, 2003.

6. FERRIGNO, I. S. V. Terapia da mão: fundamentos para a prática Clínica. São Paulo: Livraria Santos Ed., 2007.

7. FLEISHMAN, E. A.; ELLISON, G. D. A factor analysis of fine manipulative tests. J. Appl. Psychol., v. 46, n. 2, p. 96$105,1962$.

8. GARCIAS, G. L.; ROTH, M. G. M.; MESKO, G. E.; BOFF, T. A. Aspectos do desenvolvimento neuropsicomotor na síndrome de Down. Rev. Bras. Neurol., v. 31, n. 6, p. $245-$ $248,1995$.

9. GARROS, D. S. C.; GAGLIARDI, R. J.; GUZZO, R. A. R. Evaluation of performance and personal satisfaction of the patient with spastic hand after using a volar dorsal orthosis. Arq. Neuro-Psiquiatr., v. 68, n. 3, p. 385-389, 2010.

10. GIMENEZ, R.; MANOEL, E. J.; OLIVEIRA, D. L.; BASSO, L. Combinação de padrões fundamentais de movimento: crianças normais, adultos normais e adultos portadores da Síndrome de Down. Rev. Bras. Educ. Física Esporte, v. 18, n. 1, p. 101-116, 2004.

11. GODOY, J. R.; BARROS, J. F. Força de preensão palmar 
em portadores da síndrome de Down e análise dos músculos envolvidos neste movimento. Acta Cir. Bras., v. 20, supl. 1, p. 159-166, 2005.

12. GUSMAN, S; TORRE, C. A. Fisioterapia na Síndrome de Down. In: SCHWARTZMAN, J.S. (org). Síndrome de Down. São Paulo: Mackenzie-Memnom Edições Científicas, 1999.

13. GUZZO, R.A.R. Análise comparativa da medida de independência funcional verbal e vivenciada em pacientes com hemiplegia por acidente vascular cerebral. 2008. $78 \mathrm{f}$. [Dissertação] - FCMSCSP, Programa de Ciências da Saúde, São Paulo.

14. LATASH, M. L.; KANG, N; PATTERSON, D. Finger coordination in persons with Down syndrome: atypical patterns of coordination and the effects of practice. Exp. Brain Res., v. 146, n. 3, p. 345-355, 2002.

15. MAGALHÃES, L. C. Avaliação de Terapia Ocupacional: o que avaliar e como avaliar. In: CONGRESSO BRASILEIRO, 5., SIMPÓSIO LATINO AMERICANO DE TERAPIA OCUPACIONAL, 4., Belo Horizonte, 1997. Horizontes da Clínica à Pesquisa. Anais... Belo Horizonte, 1997.

16. MANCINI, M. C.; SILVA, P. C.; GONÇALVES, S. C.; MARTINS, S. M. Comparação do desempenho funcional de crianças portadoras de síndrome de Down e crianças com desenvolvimento normal aos 2 e 5 anos de idade. Arq. Neuro-Psiquiatr., v. 61, n. 2B, p. 409-415, 2003.

17. MATHIOWETZ, V.; VOLLAND, G.; KASHMAN, N.;
WEBER, K. Adult norms for the Box and Block Test of manual dexterity. Am. J. Occup. Ther., v. 39, n. 6, p. 386$391,1985$.

18. MENDES, M. F.; TILBERY, C. P.; BALSIMELLI, S.; MOREIRA, M. A.; CRUZ, A. M. B. Teste de destreza manual da caixa e blocos em indivíduos normais e em pacientes com esclerose múltipla. Arq. Neuro-Psiquiatr., v. 59, n. 4, p. 889-894, 2001.

19. MEYERHOF, P. G. O desenvolvimento normal da preensão. Rev. Bras. Cresc. Desenvolv. Hum., v. 4, n. 2, p. 25-29, 1994.

20. NUNES, C. M. P. Avaliação e técnicas de avaliação nas áreas de desempenho ocupacional. In: CAVALCANTE, A.; GALVÃO, C. R. Terapia ocupacional, fundamentação $e$ prática. Rio de Janeiro: Guanabara Koogan, 2007.

21. NUNES, M.D.R.; DUPAS, G. Independência da criança com síndrome de Down: a experiência da família. Revista LatinoAmericana de Enfermagem, v. 19, n. 4, p. 1-9, 2011.

22. OTTO, P. G.; OTTO, P. A; FROTA-PESSOA, O. Genética humana e clínica. $2^{\mathrm{a}}$ ed. São Paulo: Roca, 2004.

23. POIRIER, F. Dexterity as a valid measure of hand function: a pilot study. Occup. Ther. Health Care, v. 4, n. 3-4, p. 6983,1988 .

24. SCHWARTZMAN, J. S. Doença de Alzheimer e síndrome de Down. Temas Desenvolv., v. 1, n. 4, p.15-17, 1992.

Recebido para publicação: 05/08/2011

Aceito para publicação: 30/04/2012 